\title{
DELAYED PNEUMOTHORAX AND CONTRALATERAL HYDROTHORAX INDUCED BY A LEFT SUBCLAVIAN CENTRAL VENOUS CATHETER: A CASE REPORT
}

doi: 10.1590/S1807-59322010000500016

\author{
Nermin Kelebek Girgin, Semih Arici, Gurkan Turker, Banu Otlar, Leyla Hotaman, Oya Kutlay
}

\section{INTRODUCTION}

The placement of central venous catheters (CVCs) has become a ubiquitous practice in intensive care units (ICUs). ${ }^{1,2}$ The use of CVCs is often a necessity for the measurement of hemodynamic variables and the administration of fluids, medications, blood products, and parenteral nutrition in critically ill patients; however, catheterization may occasionally be associated with complications, such as pneumothorax, hemothorax, cardiac tamponade, sepsis, and thrombosis. ${ }^{1-4}$ These complications may be life-threatening, even when the procedure is performed under the most ideal circumstances. ${ }^{3,4}$ Although the incidence of delayed pneumothorax and hydrothorax after central venous catheterization is low, such complications of catheterization can sometimes be associated with significant morbidity and mortality. We searched Medline reports from the last 30 years and found very few cases that reported delayed pneumothorax and hydrothorax caused by catheterization. Most of these reported cases observed pneumothorax and/or hydrothorax on the same side as the CVC, $, 3,5$ and only one case developed hydrothorax as a delayed complication on the opposite side as the catheter. ${ }^{7}$ We did not detect the simultaneous occurrence of delayed pneumothorax and contralateral hydrothorax in a single case.

Here, we report a case wherein left pneumothorax and right hydrothorax were simultaneously detected as delayed complications induced by a left subclavian CVC.

Department of Anaesthesiology and Reanimation, Uludag University, School of Medicine - Bursa, Turkey

Email: ygturker@hotmail.com

Tel.: 902244428039

Fax: 902244428958

\section{CASE DESCRIPTION}

A 72-year-old female (height: $161 \mathrm{~cm}$, weight: $63 \mathrm{~kg}$ ) was admitted to the hospital with progressive and generalized muscle spasms associated with risus sardonicus and opisthotonus. Tetanus was diagnosed, and she was transferred to the intensive care unit (ICU). She was intubated and mechanically ventilated because of respiratory distress. After the intubation, a central venous catheter (CVC) (Certofix Trio, SB 730, 30-cm in length, $7^{\mathrm{F}}, 3^{\text {Lumen }}, \mathrm{B}$. Braun, Melsungen AG, Melsungen, Germany) was inserted into the left subclavian vein using the Seldinger technique. The left subclavian vein was punctured at the first attempt, and blood was freely aspirated from the vein before and after insertion of the catheter. Correct positioning of the catheter tip in the superior vena cava was confirmed by chest radiography (CXR) (Figure 1). Radiography was performed with a portable bedside machine in the antero-posterior direction with the patient lying in the supine position. The patient was sedated with intravenous diazepam and dexmedetomidine infusions.

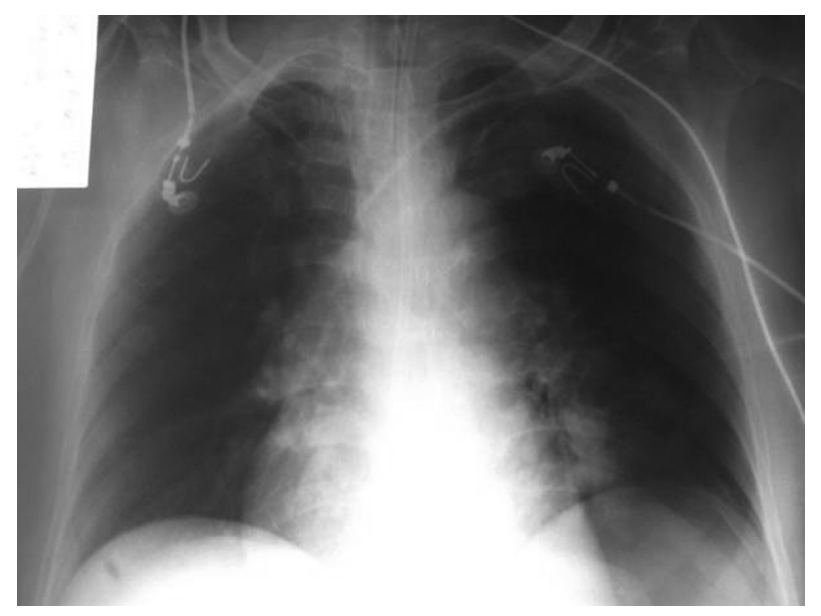

Figure 1 - Control X-ray obtained after insertion of the central venous catheter. 
Intermittent positive pressure ventilation was started as a pressure-controlled ventilation [frequency: 14 beats/min, PEEP: $3 \mathrm{cmH}_{2} \mathrm{O}$, inspiration pressure (Pins): $12 \mathrm{mmHg}$ ] (GE Healthcare, Engström Carestation). Prior the $5^{\text {th }}$ day of ICU care, no CXR was taken because the patient's hemodynamic and arterial blood gas analyses were stable. On the $5^{\text {th }}$ day, the patient developed progressive hypotension. Although the intravenous fluid infusion dose was increased, hypotension continued, and therefore, a dopamine infusion was started. In addition to hypotension, the patient began to suffer from a decrease in $\mathrm{PaO}_{2}$. Auscultation revealed that neither lung was aerated, and it was not possible to aspirate blood from the CVC ports. Therefore, a new CVC was inserted into

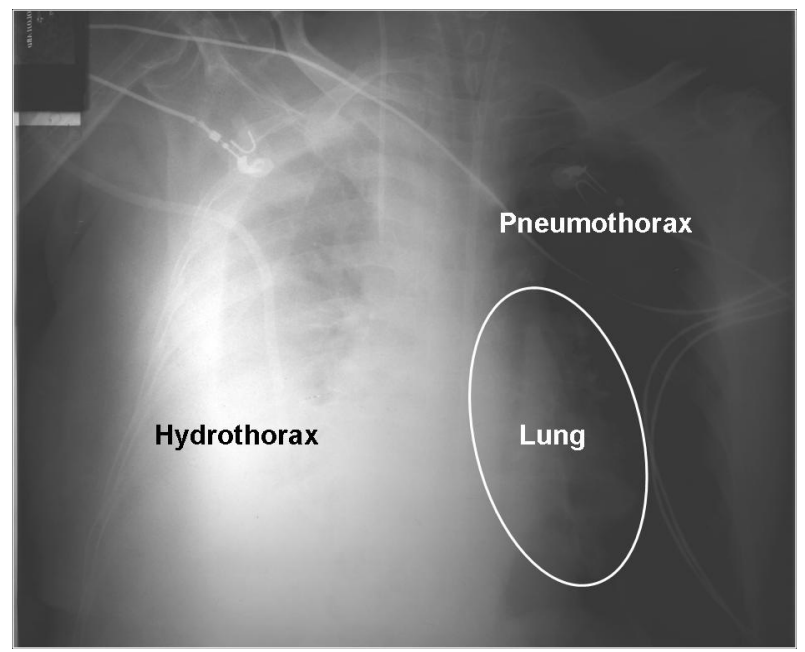

Figure 2 - Chest X-ray showing the left pneumothorax and right hydrothorax. the right internal jugular vein. CXR was then ordered, but it was not immediately performed due to technical reasons. Before a radiograph was obtained, the left subclavian CVC was removed because of a misunderstanding between experienced staff and a junior doctor. After that, CXR was performed, which detected a large left pneumothorax and a right hydrothorax (Figure 2). Bilateral thoracentesis was performed, and $2000 \mathrm{ml}$ of clear fluid was drained into the right thorax tube. This volume was comparable to the volume of fluid the patient received through the catheter. The patient's condition markedly improved, and lung expansion and gas exchange normalized (Figure 3 and Table 1). A tracheotomy was performed on the $9^{\text {th }}$ day, mechanical

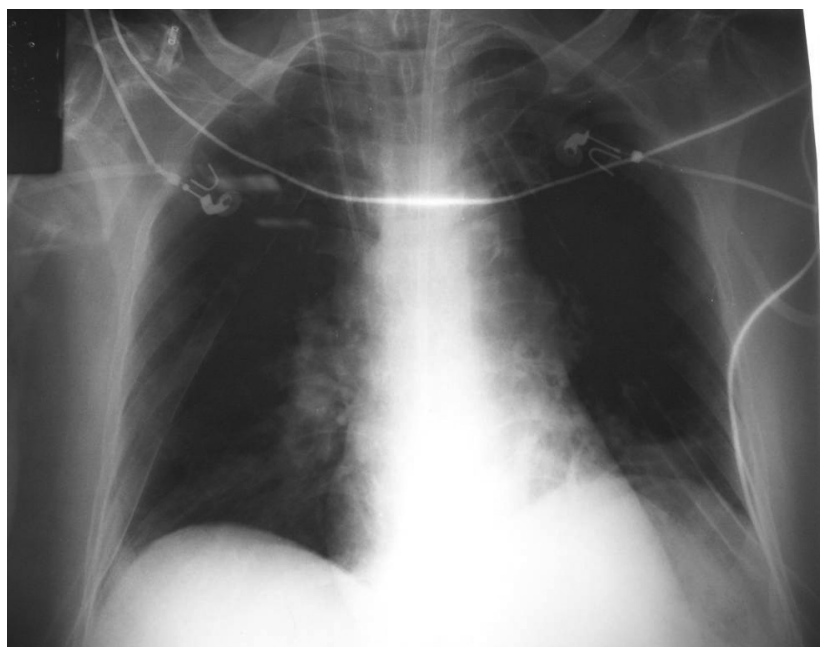

Figure 3 - Chest X-ray after bilateral thoracentesis.

Table 1 - Summary of respiratory mechanics values, artery blood gas analyses, and hemodynamic variables.

\begin{tabular}{|c|c|c|c|c|c|c|}
\hline & \multicolumn{6}{|c|}{ Time } \\
\hline & $04^{00 *}$ & $06^{00 *}$ & $12^{00 * *}$ & $13^{00} \dagger$ & $15^{00} \dagger$ & $18^{00} \dagger$ \\
\hline $\mathrm{TV}$ & 516 & 521 & 465 & 490 & 508 & 530 \\
\hline Frequency & 14 & 12 & 15 & 20 & 20 & 14 \\
\hline PEEP & 5 & 5 & 10 & 7 & 5 & 5 \\
\hline I:E & $1 / 2$ & $1 / 2$ & $1 / 2$ & $1 / 2$ & $1 / 2$ & $1 / 2$ \\
\hline Pinp & 12 & 12 & 12 & 17 & 15 & 12 \\
\hline PIP & 18 & 20 & 28 & 21 & 19 & 18 \\
\hline $\mathrm{FiO}_{2}$ & 0.5 & 0.3 & 1.0 & 1.0 & 0.5 & 0.5 \\
\hline $\mathrm{pH}$ & 7.42 & 7.39 & 7.09 & 7.16 & 7.30 & 7.36 \\
\hline $\mathrm{PO}_{2}(\mathrm{mmHg})$ & 154.5 & 89.4 & 77.4 & 258.4 & 110.2 & 137.5 \\
\hline $\mathrm{PCO}_{2}(\mathrm{mmHg})$ & 40.6 & 32.9 & 93.3 & 79.6 & 50.2 & 39.9 \\
\hline $\mathrm{BE}(\mathrm{mmol} / \mathrm{L})$ & 1.4 & -4.7 & -4.1 & -2.2 & -2.8 & -3.0 \\
\hline SAP/DAP (mmHg) & $101 / 68$ & $126 / 70$ & $91 / 52 \div$ & $132 / 81$ & $142 / 89$ & $139 / 85$ \\
\hline HR (beat $\mathrm{min}^{-}$) & 96 & 104 & 134 & 128 & 105 & 93 \\
\hline
\end{tabular}

* Before thoracentesis; $* *$ Just before thoracentesis; $\uparrow$ After bilateral thoracentesis; $\$$ with dopamine infusion (10 mcg/kg/min). TV: tidal volume; PEEP: positive end-expiratory pressure; I: inspiration; E: expiration; Pins: inspiration pressure; PIP: peak inspiratory pressure; BE: base excess; SAP: systolic arterial pressure; DAP: diastolic arterial pressure; HR: heart rate. 
ventilation was discontinued on the $34^{\text {th }}$ day, and the patient was discharged from the ICU on the $45^{\text {th }}$ day without problems.

\section{DISCUSSION}

Central venous catheterization has become an increasingly common procedure in the care of critically ill patients. It is can sometimes be associated with the immediate occurrence of acute complications during placement, such as pneumothorax and artery puncture, as well as delayed complications such as catheter-related infection and cardiac tamponade. ${ }^{1,2}$

Pneumothorax is a well-known complication of central venous catheterization using the jugular or subclavian approach. ${ }^{1}$ It can occur within days after central venous catheterization, and its diagnosis can sometimes be delayed. ${ }^{3,6}$ After the insertion of CVCs, it is currently a standard practice to confirm that the position of the catheter tip is appropriate and to eliminate radiologically detectable complications (e.g., pneumothorax and hemothorax) by $\mathrm{CXR} ;{ }^{8,9}$ however, Molgaard et al. ${ }^{8}$ investigated $473 \mathrm{CVC}$ insertion procedures, found no value in routine X-ray control after catheterization, and have since suggested that routine CXR be omitted from central venous catheterization practice. Furthermore, these authors argue that a small pneumothorax can be easily missed due to the typical poor quality of chest radiographs. Additionally, iatrogenic pneumothorax can have no initial symptoms, and moreover, a slow pleural air leak in association with positive pressure ventilation delays pneumothorax. . $^{3,6}$ Mechanical ventilatory therapy with high peak inspiratory pressure and mean airway pressure can cause barotraumasrelated iatrogenic pneumothorax. ${ }^{10}$ Lee et al. ${ }^{6}$ have also suggested that positive pressure ventilatory support in the ICU might contribute to large-sized tension pneumothorax. In our case, there was no radiological evidence from the CXR for pneumothorax immediately after CVC insertion, and furthermore, the patient's peak inspiratory pressure was acceptable during the treatment period. Therefore, we thought that we may have missed a pneumothorax on the first CXR because of the poor quality of chest radiography or because the pneumothorax was too small to detect; however, we could not explain the observed contralateral hydrothorax based on these mechanisms.

Hydrothorax is another complication of central venous catheterization. ${ }^{3,5,7}$ It can develop if part or all of the catheter is removed from the vein or perforates the vein while continuing the infusion of fluid. Perforation is thought to occur as a result of mechanical trauma from the catheter tip or chemical damage from infused solutions.${ }^{11}$ The angle that the catheter tip forms with the wall of a vein is thought to be an important factor that is responsible for vessel trauma. When the tip lies at a more perpendicular angle to the wall, there is an increased chance of direct vessel trauma and erosion. Left-sided catheters pose a particular problem because the left innominate vein forms a nearly right angle with the superior vena cava (SVC). Catheters entering the SVC from the left therefore have a tendency to impinge on the lateral wall of the SVC. ${ }^{12}$ Unfortunately, CXR may not show any abnormalities until considerable fluid has accumulated in the pleura. ${ }^{13}$ Although hydrothorax has been occasionally described, delayed contralateral hydrothorax is quite rare. Colón and Frazier ${ }^{7}$ have described a patient with a right hydrothorax as a delayed complication after left subclavian vein catheterization, wherein they suggested that the position of the catheter was the primary causative factor of the observed contralateral hydrothorax. These authors have suggested that the location where the catheter was placed is an area of turbulent flow due to the convergence of two vessels and to the azygos vein that joins the superior vena cava posteriorly. Thus, the tip of the catheter, which lies close to the lateral wall of the superior vena cava, creates the risk of wall erosion in the presence of turbulent flow. Erosion may lead to perforation of the vein, and fluids may then be directly infused into the right pleural cavity. ${ }^{13}$ These authors have suggested that this complication could most likely be prevented by optimal positioning of the CVC when using the left-sided approach.

In addition to the catheter tip's position, catheter tip migration can also be responsible for contralateral hydrothorax. Catheter tip migration is a well-recognized phenomenon that occurs after central venous cannulation in up to $17 \%$ of percutaneously introduced catheters. ${ }^{3,14}$ In adults, the tip of a catheter inserted via the subclavian or internal jugular vein can move by up to $2 \mathrm{~cm} .{ }^{13}$ The extrusion of central lines in multilumen catheters can be caused by inadequate fixation of the catheters and/or movements of the patient that result in extravascular positioning of at least one lumen. It has been suggested that pleural effusions and mediastinal widening may also occur via this mechanism. ${ }^{15}$ The injection of radiographic contrast provides a useful method for investigating the diagnosis of extravasation or aberrant CVC placement. ${ }^{13-15}$

In addition to catheter tip position and migration, the site of insertion is an important factor. Subclavian catheterization is more likely to be complicated by pneumothorax and hemothorax than internal jugular catheterization. ${ }^{1}$ Catheters in the subclavian vein (especially the left) and left internal jugular vein cause concern because of the more perpendicular angle with which the tip may lie relative to the vessel wall. ${ }^{13}$ In our case, the catheter was placed via the left subclavian vein. 
To the best of our knowledge, this is the first report to describe a case of unilateral pneumothorax on the same side as the left CVC and a unilateral hydrothorax on the opposite side of the CVC. In this case, there were no clinical signs (e.g., hypotension or tachypnea) of pneumothorax or hydrothorax until 5 days after CVC insertion. Therefore, a high index of suspicion should be maintained, especially in patients receiving positive pressure ventilation and deep sedation, as was the case for our patient. It should be kept in mind that complications resulting from central venous catheterization can occur at later stages as well. This case emphasized the need to carefully monitor the patient's vital signs and symptoms in order to detect and effectively treat the rare but potentially lethal complications of central venous catheterization. Additionally, the preferential use of rightsided central access should be considered where possible.

\section{REFERENCES}

1. McGee DC, Gould MK. Preventing complications of central venous catheterization. N Eng J Med. 2003;348:1123-33.

2. Merrer J, De Jonghe B, Golliot F, Lefrant JY, Raffy B, Barre E, et al. Complications of femoral and subclavian venous catheterization in critically ill patients: a randomized controlled trial. JAMA. 2001;286:700-7.

3. Thomas CJ, Butler CS. Delayed pneumothorax and hydrothorax with central catheter migration. Anaesthesia. 1999;54:987-8.

4. Krauss D, Schmidt GA. Cardiac tamponade and contralateral hemothorax after subclavian vein catheterization. Chest. 1991;99:517-8.

5. Maisniemi KJ, Koljonen VS. Tension hydrothorax induced by central venous catheter migration in a patient with burn. Br J Anaesth.2002;89:647-50.

6. Lee SH, Lee JW, Sohn JT, Lee HM, Shin IW, Lee HK, et al. Delayed tension pneumothorax detected 4 days after central venous catheterization. Korean J Anesthesiol. 2008;54:59-61.

7. Colon R, Frazier OH. Right hydrothorax after left subclavian and internal jugular vein catheterization: A delayed complication. Tex Heart Inst J. 1985; $12: 389-92$.

8. Molgaard O, Nielsen MS, Handberg BB, Jensen JM, Kjaergaard J, Juul N. Routine X-ray control of upper central venous lines: Is it necessary? Acta Anaesthesiol Scand. 2004;48:685-9.
9. Bailey SH, Shapiro SB, Mone MC, Saffle JR, Morris SE, Barton RG. Is immediate chest radiograph necessary after central venous catheter placement in a surgical intensive care unit? Am J Surg. 2000;180:517-21.

10. Celik B, Sahin E, Nadir A, Kaptanoglu M. Iatrogenic pneumothorax: etiology, incidence and risk factors. Thorac Cardiovasc Surg. 2009;57:286-90.

11. Flatley ME, Schapira RM. Hydropneumomediastinum and bilateral hydropneumothorax as delayed complications of central venous catheterization. Chest. 1993;103:1914-6.

12. Fletcher SJ, Bodenham AR. Safe placement of central venous catheters: where should the tip of the catheter lie? Br J Anaesth. 2001;87:298-302.

13. Booth SA, Norton B, Mulvey DA. Central venous catheterization and fatal cardiac tamponade. Br J Anaesth. 2001;87:298-302.

14. Paw HG. Bilateral pleural effusions: unexpected complication after left internal jugular venous catheterization for total parenteral nutrition. $\mathrm{Br}$ J Anaesth. 2002;89: 647-50.

15. Bach A. Complications of central venous catheterization. Chest. 1993;104:654-5. 